\title{
An Efficient Non-Preemptive Algorithm for Soft Real-Time Systems using Domain Cluster-Group EDF
}

\author{
R. Kalpana \\ Dept. of CSE \\ Pondicherry Engg. College
}

\author{
S. Keerthika \\ Dept. of CSE \\ Pondicherry Engg. College
}

\begin{abstract}
In this paper a new non-preemptive domain clustering scheduling algorithm for soft real time application is proposed. The main aim of this algorithm is to achieve high deadline meeting ratio of the group Earliest Deadline First (gEDF) algorithm by maintaining the excellent performance during normal load. An efficient non-preemptive algorithm called Domain Cluster - group EDF (DC-gEDF) is proposed for real time systems which makes clustering the task according to the domain specification with their deadlines and schedules the tasks within a group. The results are analysed and compared for the metric deadline meeting ratio of gEDF and DC-gEDF under different deadline acceptable values. It shows an improvement in the deadline meeting ratio for the proposed DC-g-EDF algorithm.
\end{abstract}

\section{General Terms}

Real time scheduling

\section{Keywords}

Group scheduling, EDF, group EDF, soft real time, nonpreemptive

\section{INTRODUCTION}

Earliest deadline first (EDF)is a dynamic scheduling algorithm [5] used in real-time task scheduler to place the tasks in a priority queue [19]. Whenever a scheduling processes need to perform the queue will be searched for the task closest to its deadline. This task is to be scheduled for next execution [2], [4]. EDF is an optimal scheduling algorithm on preemptive scheduling processors, in that sense: if a collection of independent tasks, each characterized by an start time, an requirement and a deadline, can be scheduled by any algorithm in a way that ensures all the tasks complete by their deadline, the EDF will schedule this collection of tasks so they all complete by their deadline.

In preemptive systems, tasks are pre-empted by higher priority tasks or by some other techniques, while nonpreemptive systems will not permit pre-emption. Preemptive scheduling algorithms are easy to develop but non-preemptive scheduling algorithms are more efficient than the preemptive. However, non-preemptive EDF techniques have produced near optimal schedules for periodic and aperiodic tasks, particularly when the system is lightly loaded [3]. When the system is overloaded, it has been shown that the EDF approach leads to very poor performance (i.e., low success rates). In this dissertation, a system load or utilization is used to refer to the ratio of the sum of the execution times of pending tasks and the time available to complete the tasks [9], [12]. The reason for the poor performance of EDF is because of tasks that are scheduled based on their deadlines, sometimes they miss their deadlines due to overload, so that other tasks waiting for their turn for long time so their deadlines are also have the chance to miss also - it is sometime known as the domino effect. To overcome these issues, the derivation of EDF algorithm is used. It is group EDF (gEDF), where the tasks with "similar" deadlines are grouped together (i.e., deadlines that are very close to one another), and the Shortest Task First (SJF) algorithm is used for scheduling tasks within a group. It should be noted that our approach is different from adaptive schemes that switch between different scheduling strategies based on system load. gEDF is used in overloaded as well as under loaded conditions.

However gEDF produces efficient scheduling results, it does not concerns domain specification of tasks. Our approach is to analyze the specification of given tasks and grouping them with similar one using gEDF and with new scheduling algorithm called Domain Cluster - group EDF (DC - gEDF). This algorithm groups the tasks according to the domain and applies gEDF to each group. So that each group can schedule simultaneously, this reduces the overall duration to complete the tasks.

\section{RELATED WORK}

\subsection{Shortest Task First (SJF)}

Shortest Task First (SJF) scheduling is probably optimal but requires clairvoyance, profiling, or expected execution time to fully implement. SJF can be implemented either preemptively or non-pre-emptively. SJF has low average waiting time [10], [11], [20]. In fact, SJF is optimal with respect to average waiting time. It is very easy to prove this claim by comparing it with other real-time algorithms.

In Soft Real Time systems [13], [14], each task may have one or more than one predecessors. SJF algorithm [7] cannot analyze the predecessors of the tasks. To overcome this issue another real time scheduling algorithm has been proposed, called Earliest Deadline First (EDF). 


\subsection{Earliest Deadline First (EDF)}

Each task in an EDF scheduler is assigned with a deadline. Every time a task is inserted in the system, the scheduling system looks for the other task which is present in the queue and which has the next nearest deadline and select it for execution [7]. In order to ensure that the scheduling application is still able to meet each deadline, a 'scheduler must evaluate if each new incoming task doesn't overload the system and slow down the execution if it will do so.

However, EDF[8] performs worst during overload (i.e. when the total CPU utilization exceeds $100 \%$ ) condition [4], [15]. This poor performance may create the chance to miss the deadline of the tasks. And this leads that other tasks need to wait for a turn longtime and miss their deadline too.

\subsection{Group Earliest Deadline First (gEDF)}

In this concept the deadlines are formed as a group according to their range of deadlines, dynamically. Here groups are separated by the concept of traditional EDF but the group may be arranged by different techniques say priority or SJF value, etc. We use SJF to develop EDF, but it is one of the techniques among the scheduling methods. gEDF is mainly suitable for non-preemptive soft-real-time systems.

group in the gEDF algorithm depends on a set of deadline group range that is, these are grouped according to the deadlines named as parameter Gr. A task $\tau$ jbelongs to the same group as task $\tau i$ if $\operatorname{di} \leq \mathrm{dj} \leq(\mathrm{di}+\mathrm{Gr} *(\mathrm{di}-\mathrm{ti}))$, where ti is the current time, $1 \leq \mathrm{i}, \mathrm{j} \leq \mathrm{N}$ [1]. We group tasks with deadlines which are close to each other. The tasks with close deadlines are in a group.

We assume a uniprocessor system [16]. QgEDF is a queue for gEDF scheduling. The current time is represented by $t$. QgEDF| represents the length of the queue QgEDF. $\tau=(\mathrm{r}, \mathrm{e}$, $\mathrm{D}, \mathrm{P})$ is the task at the head of the queue.

- gEDF Group $=\{\tau \mathrm{k} \mid \tau \mathrm{k} \in$ QgEDF, dik - di $1 \leq \mathrm{D} 1 *$ Gr, 1 $\leq \mathrm{k} \leq \mathrm{m}$, where $\mathrm{m} \leq|\mathrm{QgEDF}|\}$, and $\mathrm{D} 1$ is the deadline of the first task in a group

The pseudo code of the functions Enqueue and Dequeue are given below:

Function Enqueue $\left(\mathbf{Q}_{\mathrm{gEDF}}, \tau\right)$

if ( $\tau$ 's deadline $d>t i$ ) then inserttask $\tau$ into $\mathrm{Q}_{\mathrm{gEDF}}$ by Earliest Deadline First, i.e. $d_{i} \leq d i_{i+1} \leq d i_{i+2}$, where $\tau_{i}$, end $\tau_{i+1}, \tau_{i+2} \in \mathrm{Q}_{\mathrm{gEDF}}, 1 \leq i \leq\left|\mathrm{Q}_{\mathrm{gEDF}}\right|-2$;

- Enqueue is called on eachtask arrivals.

Function Dequeue $\left(\mathbf{Q}_{\mathrm{gEDF}}\right)$

if $\mathrm{Q}_{\mathrm{gEDF}} \neq \phi$ then

find a task $\tau_{\min }$ with $e_{\min }=\min \left\{e_{k} \mid \tau_{k} \in\right.$ $\mathrm{Q}_{\mathrm{gEDF}}, d i_{k}-d i_{1} \leq G r * D_{l}, 1 \leq k \leq m$, where $\left.m \leq\left|\mathrm{Q}_{\mathrm{gEDF}}\right|\right\}$;run it and delete $\tau_{\text {min }}$ from $\mathrm{Q}_{\mathrm{gEDF}}$;

end

- Dequeue is called when the execution of all task ends.

\section{DOMAIN CLUSTER - GROUP EDF (DC-G-EDF)}

\section{A. Definitions}

To define our algorithm we use the following notations.

$\tau i$ - denotes task $i$

$D i-$ absolute deadline of task $i$

$d i$ - dynamic deadline of task $i$
$U t$ - denotes total cpu utilization

$T r$-denotes the deadline tolerance of soft real time system

$e i-$ denotes the execution time of task $i$

B. Scheduling Model: Non-Preemptive Real Time

Non-preemptive scheduling [17], [18] is much efficient than preemptive scheduling since preemption requires context switching method overhead which may be significant in wellformed multithreading systems. In this non-preemptive model SJF algorithm is used to schedule the tasks according to the deadlines (i.e. which has small deadlines that are arranges first).

\section{The Algorithm}

We first group the tasks with their domain specification together based on our novel algorithm. Within the group the tasks were sorted based on another algorithm, where the task at the head of the queue will be executed first followed by the rest of the tasks in the queue sequence. A group member in the Domain cluster group is to be rescheduled according to the predecessors. And then, have to apply the gEDF algorithm to schedule finally.

The clustering is based on the domain using the following algorithm

Function Domain-Cluster ( $\left.\mathbf{Q}_{\mathrm{DCgEDF}}, \mathrm{DC}\right)$

for $\mathrm{i}=1$ to $\mathrm{i}<=\left|\mathrm{Q}_{\mathrm{DC}}\right|\{$

for $\mathrm{j}=\mathrm{i}+1$ to $\mathrm{j}<=\left|\mathrm{Q}_{\mathrm{DC}}\right|$

findDC $C_{\mathrm{i}}$ which has same domain name and put it in QDCgEDF;

//gEDF with predecessor constraints

ID2: for $\mathrm{i}=1 \mathrm{TO} \mathrm{i}<=\left|\mathrm{Q}_{\mathrm{DCgEDF}}\right|\{$

$\mathrm{T}_{\mathrm{DCgEDF}}=\mathrm{Q}_{\mathrm{DCgEDF}}$

If $(\operatorname{Pr}[\tau i] !==0)$ then

$\mathrm{C}_{\mathrm{DCgEDF}}=\mathrm{T}_{\mathrm{DCgEDF}}$

If ( flag $[\tau i]==0)$ then ID1: if $(m !==0)$ then

For $\mathrm{j}=1$ to $\mathrm{j}<=\operatorname{Pr}[\tau \mathrm{i}]\{$ $\mathrm{Sub}[\tau \mathrm{i}]=\mathrm{S}[\mathrm{T}[\tau \mathrm{ij}]$;

if $($ flag $[\operatorname{Sub}[\tau i]]==0) \quad$ then $\quad \mathrm{T}_{\mathrm{DCgEDF}}=$ Sub[ $\tau$ i $]$; m++; goto ID1;

end $\quad\}$

end

end

print $\tau$ i from $\mathrm{Q}_{\mathrm{DCgEDF}}$

\}

\section{NUMERICAL RESULTS}

The following Table 1 depicts the resources, predecessor associated with each domain along with the time (duration) taken for each activity.

Table 1.Resource allocation

\begin{tabular}{|c|c|c|c|c|}
\hline Activity & Domain & Resources & Predecessor & $\begin{array}{c}\text { Time( } \\
\text { days) }\end{array}$ \\
\hline A & D1 & 3 & 0 & 2 \\
\hline B & D2 & 3 & 0 & 3 \\
\hline C & D1 & 3 & A & 2 \\
\hline D & D2 & 2 & A,B & 4 \\
\hline E & D3 & 1 & C & 4 \\
\hline F & D1 & 4 & C & 3 \\
\hline G & D1 & 4 & D,E & 5 \\
\hline H & D2 & 2 & F,G & 2 \\
\hline
\end{tabular}


The following graph in Fig. 1 depicts the dependency graph for the activities tabulated above:

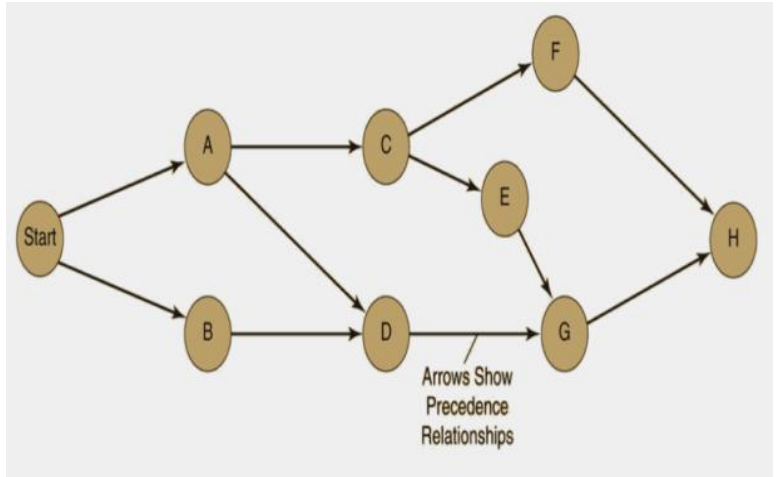

1. Some activities can be done simultaneously so project duration should be less than 49 days.

2. Critical path analysis is used to determine project duration.

3. The critical path is the longest path through the network.

\section{Domain Clusters}

Now here in Fig. 2, this process has been formed into three clusters according to their Domain specification. (i.e.) Domain 1 (D1) consists of processes A, C, F and G. Domain 2 (D2) consists of processes B, D and H. Finally Domain 3 (D3) consists of process E. So have to consider each cluster for Critical Path analysis and have to apply gEDF to each cluster. Using EDF and gEDF algorithm we couldn't do the domain analysis.

Fig 1: Determining the Project Schedule

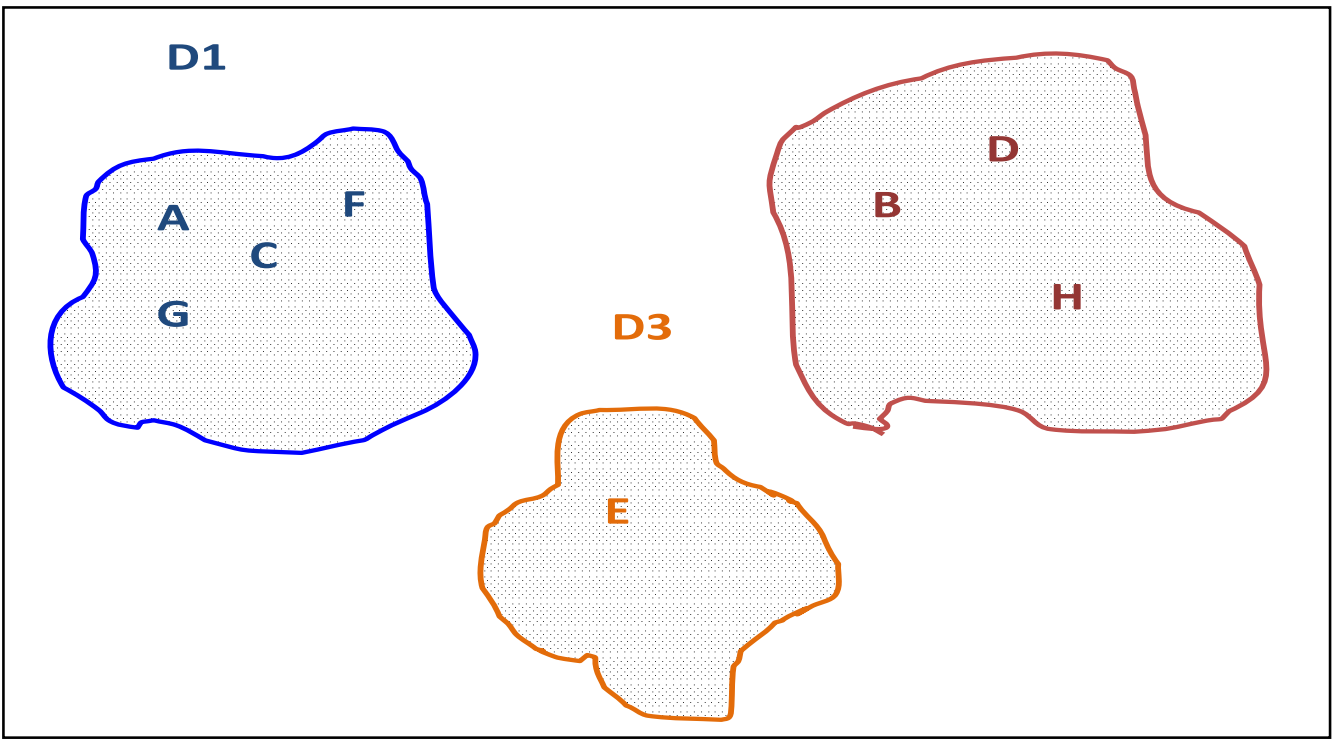

Fig 2: Representation of Domain Clusters

Without Domain analysis we cannot able to schedule the process to the members efficiently. If processes are scheduled to the members randomly, there is a chance of misleading and which results the projects may not be completed within scheduled duration. So this DC-gEDF algorithm possesses domain clustering to schedule the projects to the members according to the specification. Domain clustering analysis took place for assignment of project and not for scheduling.

\section{Critical Path Analysis (CPA)}

For CPA analysis, have to find the following values:

$$
\begin{array}{ll}
1 & \text { Earliest Start Time (EST) } \\
2 & \text { Earliest Finish Time (EFT) } \\
3 & \text { Latest start time (LST) } \\
4 & \text { Latest Finish Time (LFT) }
\end{array}
$$

\section{EFT Rule states that} $\mathrm{EFT}=\mathrm{EST}+$ activity time

Node Notation: The node notation in Fig. 3 showing the EST, EFT, LST and LFT is as follows:

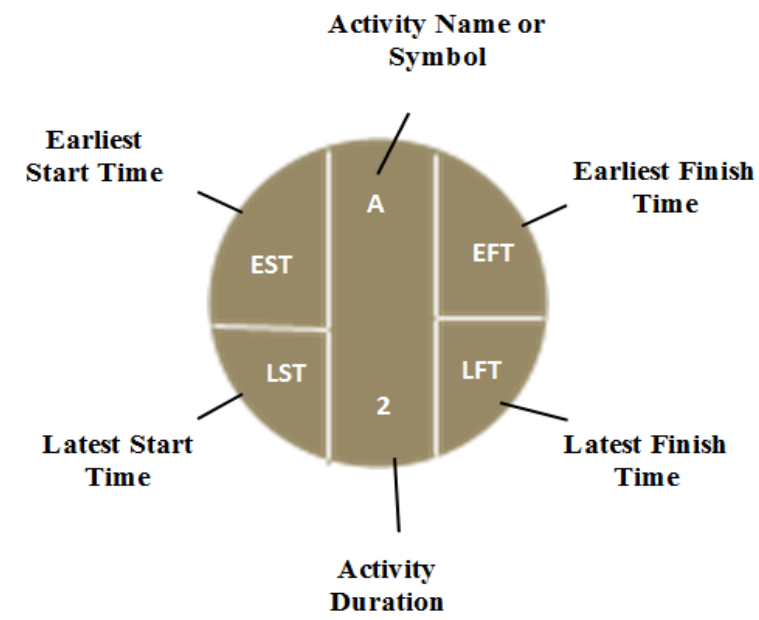

Fig 3: Notation of a Node 


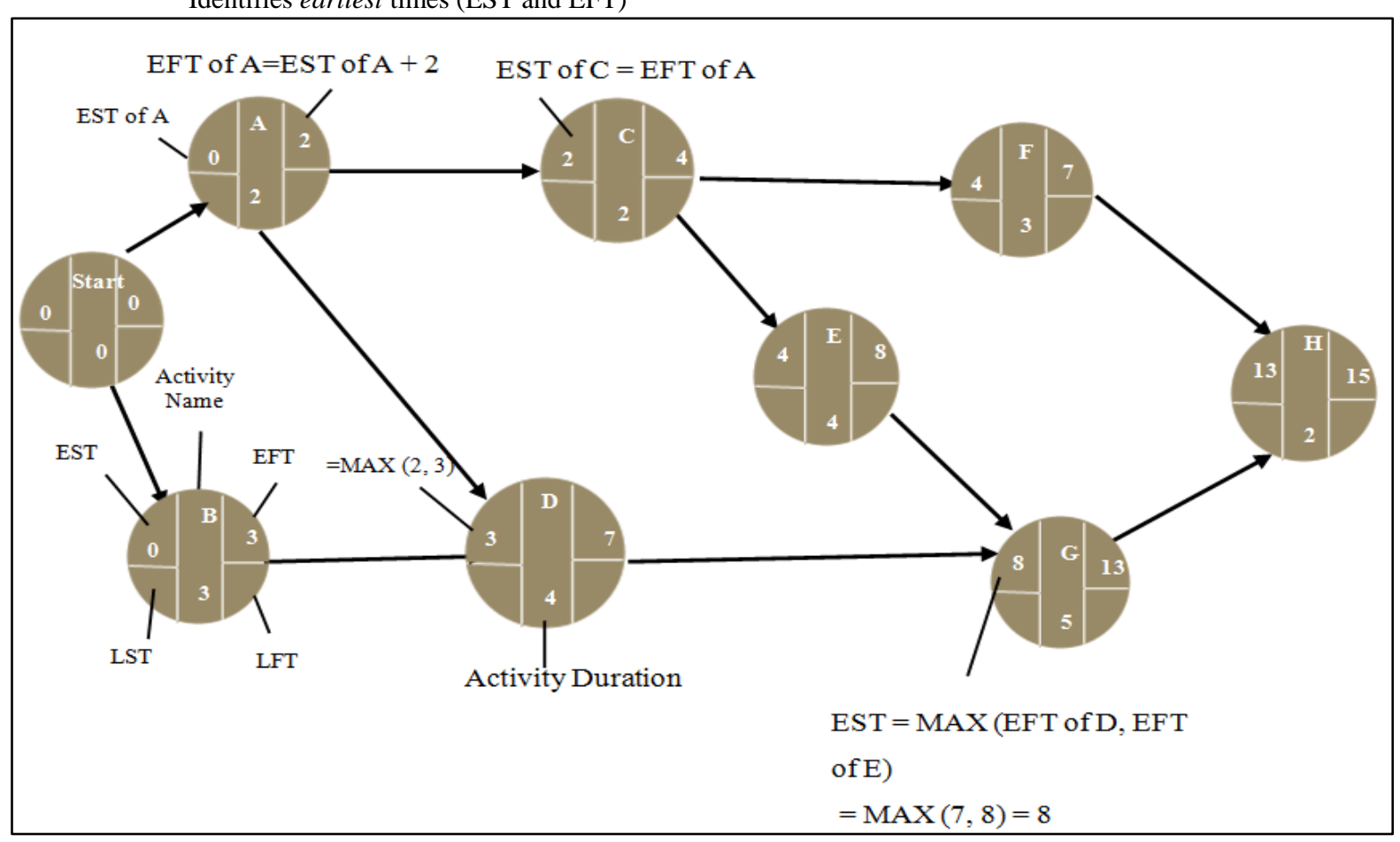

Fig 4: Forward Pass

- If there one immediate predecessor, then $\mathrm{EST}=\mathrm{EFT}$ of predecessor

Backward Pass

If $>1$ immediate predecessors, then

The pseudo code for the backward pass is shown below:

1. Identifies latest times (LST an LFT)

2. LFT Rule:
a. If activity is the immediate predecessor to only 1 activity, then
b. $\quad$ LFT $=$ LST of immediate follower
c. If activity is the immediate predecessor to multiple activities, then
d. $\quad$ LFT $=$ Min $\{$ LST of all immediate followers

$\mathrm{EST}=\mathrm{MAX}\{$ EFT's of all predecessor $\}$

Forward Pass: The forward pass is based on the Earliest Start and Finish Times, which is given in Fig. 4.

\section{LST Rule:}

$$
\text { LST }=\text { LFT }- \text { activity time }
$$

Backward Pass: The backward pass is based on Latest Start and Finish Times which is given in Fig. 5. 


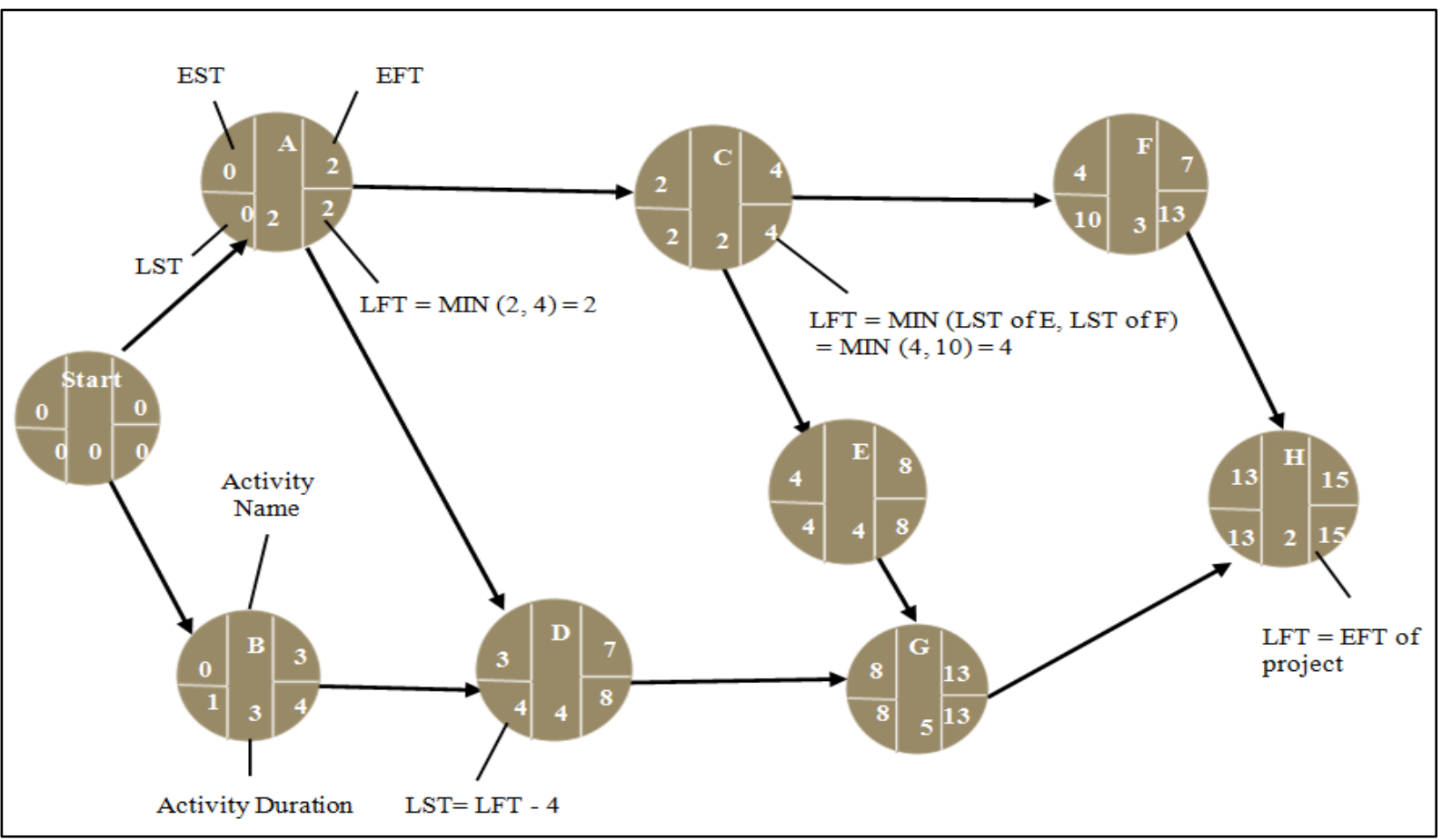

Fig 5: Backward Pass

\section{Slack Time and Critical Path(s)}

1. Slack is the total duration of an activity which may be delayed without delaying the overall project

$$
\text { i. Slack }=\text { LST }- \text { EST }
$$

2. Activities which have slack 0 are CriticalActivities
3. The Critical Path is a continuous path throughout the project from start to endwhich includes only critical activities

Project Schedule and Slack Times

The following Table 2 shows the EST, EFT, LST, LFT, Slack time corresponding to every activity. In addition to it, whether an activity is a part of critical path is also shown in Fig. 6 .

Table 2. Project Schedule

\begin{tabular}{|c|c|c|c|c|c|c|}
\hline ACTIVITY & $\begin{array}{c}\text { ES } \\
\text { T }\end{array}$ & $\begin{array}{c}\text { EF } \\
\text { T }\end{array}$ & $\begin{array}{c}\text { LS } \\
\text { T }\end{array}$ & $\begin{array}{c}\text { LF } \\
\text { T }\end{array}$ & $\begin{array}{c}\text { SLA } \\
\text { CK, } \\
\text { LST- } \\
\text { EST }\end{array}$ & ON CRITICAL PATH? \\
\hline A & 0 & 2 & 0 & 2 & 0 & Yes \\
\hline B & 0 & 3 & 1 & 4 & 1 & No \\
\hline C & 2 & 4 & 2 & 4 & 0 & Yes \\
\hline D & 3 & 7 & 4 & 8 & 1 & No \\
\hline E & 4 & 8 & 4 & 8 & 0 & Yes \\
\hline F & 4 & 7 & 10 & 13 & 6 & No \\
\hline G & 8 & 13 & 8 & 13 & 0 & Yes \\
\hline H & 13 & 15 & 13 & 15 & 0 & Yes \\
\hline
\end{tabular}




\section{Critical Path and Slack Times}

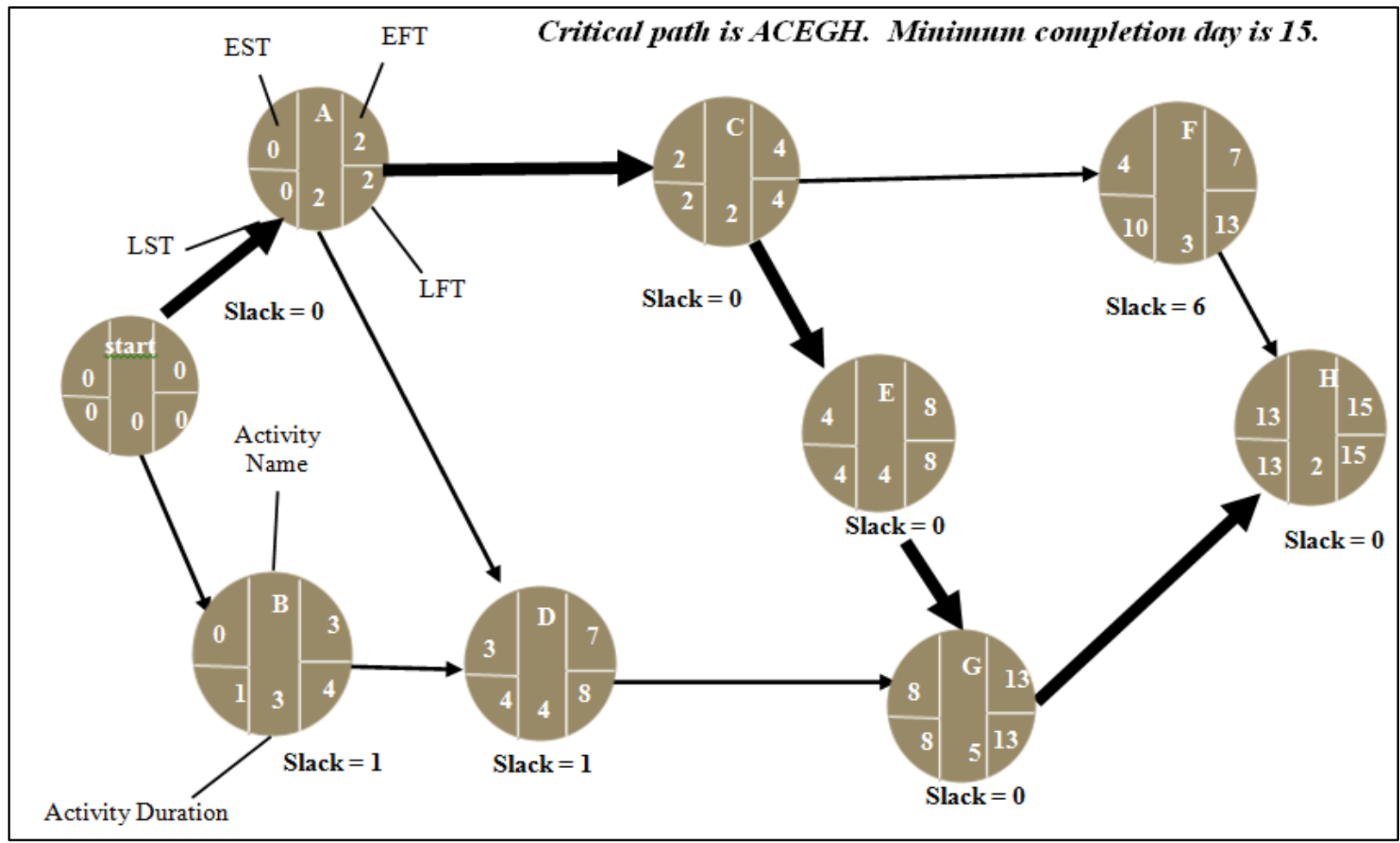

Fig 6. Critical in the schedule

\section{GANTT CHART}

The Gantt chart for all the activities involved is depicted below in Fig. 7:

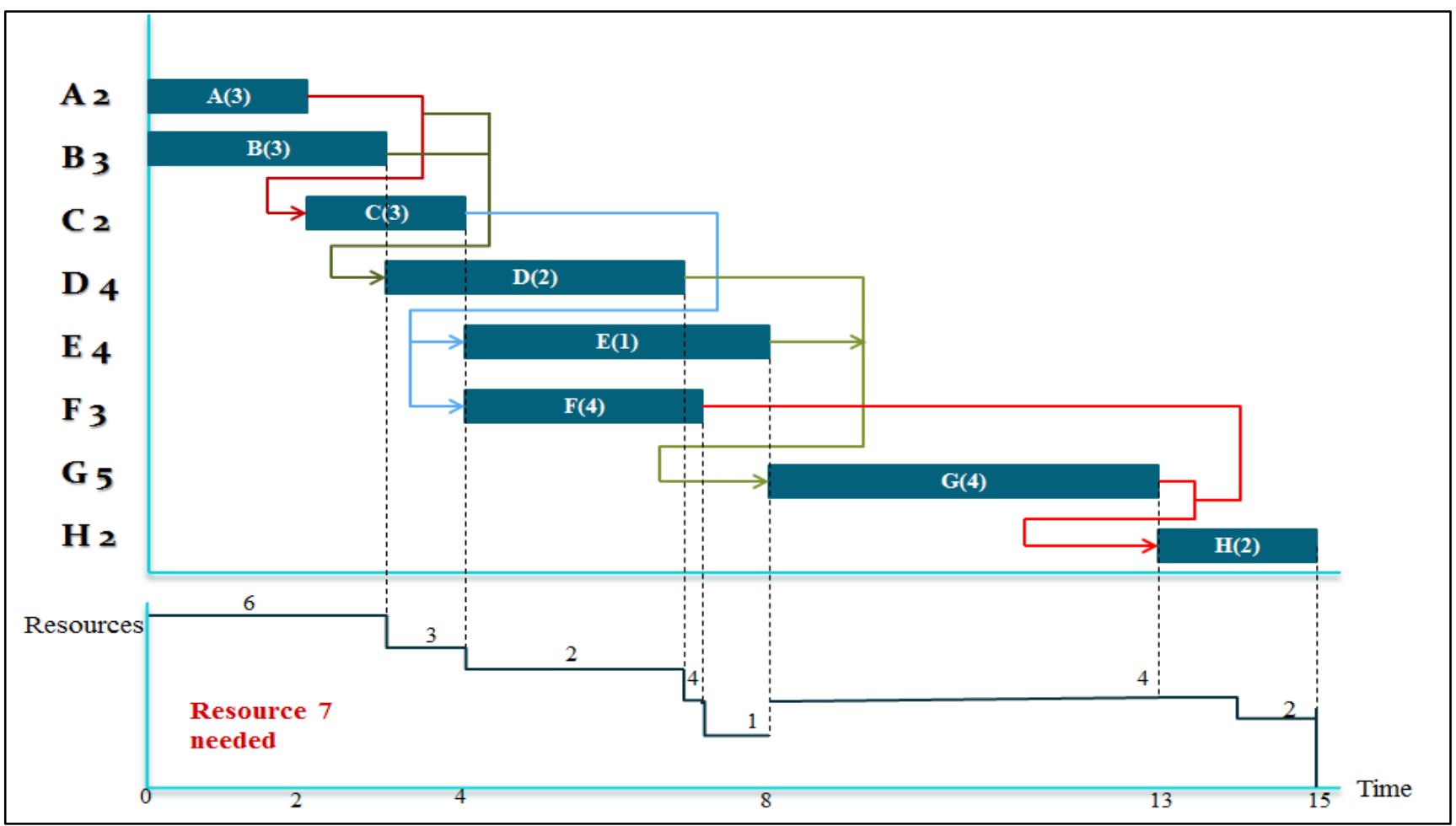

Fig 7. Gantt chart for activities

In this Gantt chart the clear work schedule has been defined. The process $A(D 1)$ and $B(D 2)$ has no predecessors so they are scheduled first. Then C (D1) has been scheduled followed by $\mathrm{D}(\mathrm{D} 2)$ is scheduled. Now $\mathrm{E}(\mathrm{D} 3)$ and $F(\mathrm{D} 1)$ are scheduled concurrently because both are dependent on and completed before itself. Then $G(D 1)$ and H(D2) are scheduled. So total time taken to complete this project will 15 days and the resource needed for this allocation is 7 Units. 


\section{Effect of Deadline Tolerance on Deadline Meeting Rate}

Comparison between EDF, gEDF and DC - gEDF DMTR

The below figure Fig. 8 shows the deadline meeting rate of EDF, gEDF and DC - gEDF algorithm as Tr in $100 \%$. We observe that DC - gEDF achieve a much better DMTR.

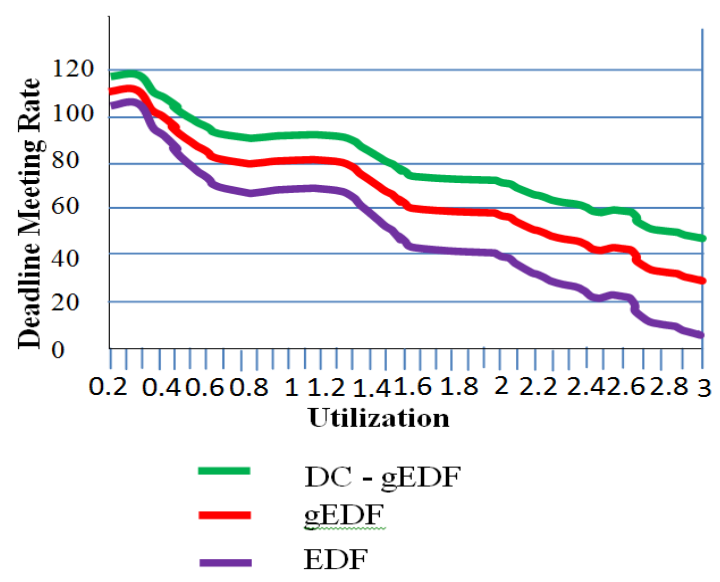

Fig. 8. Effect of Deadline Tolerance

From the above figure, it clearly shows that DC- gEDF has produce much better DMTR during normal load and has shown very significant increment during overload as the deadline tolerance value increases. And it clearly states that duration of a project will be much lower than EDF and gEDF because DC - gEDF has high deadline meeting rate. It never misses the deadline.

\section{Comparisonof EDF, g-EDF And DC-g- EDF}

The comparison between Earliest Deadline First, Group Earliest Deadline First and Domain Clustering- Group Earliest Deadline first is tabulated in Table 3.

Table 3. Comparison of EDF, g-EDF and DC-g-EDF

\begin{tabular}{|c|c|c|}
\hline EDF & GEDF & DC-GEDF \\
\hline $\begin{array}{c}\text { Pre-emptive } \\
\text { scheduling }\end{array}$ & $\begin{array}{c}\text { Non-preemptive } \\
\text { scheduling }\end{array}$ & $\begin{array}{c}\text { Domain specified } \\
\text { non-preemptive } \\
\text { scheduling }\end{array}$ \\
\hline $\begin{array}{c}\text { No predecessor } \\
\text { constraints }\end{array}$ & $\begin{array}{c}\text { No predecessor } \\
\text { constraints }\end{array}$ & $\begin{array}{c}\text { Solved with } \\
\text { predecessor } \\
\text { constraints }\end{array}$ \\
\hline $\begin{array}{c}\text { No group formed } \\
\text { chance to miss the } \\
\text { deadlines of the } \\
\text { tasks when the } \\
\text { system is } \\
\text { overloaded. }\end{array}$ & $\begin{array}{c}\text { Group based on } \\
\text { small units of } \\
\text { deadlines }\end{array}$ & $\begin{array}{c}\text { Group based on both } \\
\text { domain and } \\
\text { no chance to miss } \\
\text { the deadlines. }\end{array}$ \\
\hline $\begin{array}{c}\text { Even small } \\
\text { process need to } \\
\text { wait long for its } \\
\text { turn. }\end{array}$ & $\begin{array}{c}\text { Deadline groups are } \\
\text { scheduled } \\
\text { independently. }\end{array}$ & $\begin{array}{c}\text { Efficient then both } \\
\text { EDF } \text { gEDF. }\end{array}$ \\
\hline $\begin{array}{c}\text { Scheduling order } \\
\text { will not be } \\
\text { efficient. }\end{array}$ & $\begin{array}{c}\text { It is efficient. } \\
\text { However, there is no } \\
\text { proper domain } \\
\text { matching process. }\end{array}$ & $\begin{array}{c}\text { It solved both the } \\
\text { problem. }\end{array}$ \\
\hline \multicolumn{2}{|c|}{ independently. } \\
\hline
\end{tabular}

\section{CONCLUSION}

Non-preemptive scheduling is more efficient for soft real time systems where in context switching overhead is totally eliminated. As, the Earliest Deadline First scheduling algorithm is based on preemptive scheduling, it works fine only when the system is slightly loaded. Group Earliest Deadline First algorithm is based on non-preemptive scheduling solves this problem by grouping tasks with the same deadlines to be met. However, Group Earliest Deadline First algorithm doesn't consider the tasks in a domain-specific manner. As proposed in this paper, Domain Clustering Group Earliest Deadline First (DC-gEDF), groups tasks considering their domain along with their deadlines which eliminates the drawback in gEDF. Moreover, gEDF had no predecessor constraints. But DC-gEDF includes the predecessor constraints and is thereby more efficient than EDF and gEDF.

\section{REFERENCES}

[1] Wenming Li, B.S., M.S., GROUP EDF - A NEW APPROACH AND AN EFFICIENT NONPREEMPTIVE ALGORITHM FOR SOFT REAL-TIME SYSTEMS, UNIVERSITY OF NORTH TEXAS, August 2006.

[2] R. Jain, C. J. Hughes, and S. V. Adve, "Soft Real-Time Scheduling on Simultaneous Multithreaded Processors", In Proceedings of the $23^{\text {rd }}$ IEEE International Real-Time Systems Symposium, December 2002.

[3] K. Jeffay and C. U. Martel, "On Non-Preemptive Scheduling of Periodic and Sporadic Tasks", Proceedings of the 12 IEEE Real-Time Systems Symposium, San Antonio, Texas, December 1991, IEEE Computer Society Press, pp. 129-139.

[4] C. D. Locke, "Best-Effort Decision Making for RealTime Scheduling", CMU-CS-86-134 (PhD Thesis), Computer Science Department, Carnegie-Mellon University, 1986.

[5] S. Zilberstein, "Using Anytime Algorithms in Intelligent Systems", AI Magazine, fall 1996, pp.71-83.

[6] R. Heckmann, M. Langenbach, S. Thesing, and R. Wilhelm, "The Influence of Processor Architecture on the Design and the Results of WCET Tools", Proceedings of IEEE July 2003, Special Issue on Realtime Systems.

[7] J. Nieh and M. S. Lam, "A SMART Scheduler for Multimedia Applications", ACM Transactions on Computer Systems, Vol. 21, No. 2, May 2003.

[8] S. K. Baruah and J. R. Haritsa, "Scheduling for Overload in Real-Time Systems", IEEE Transactions on Computers, Vol. 46, No. 9, September 1997.

[9] B. D. Doytchinov, J. P. Lehoczky, and S. E. Shreve, "Real-Time Queues in Heavy Traffic with EarliestDeadline-First Queue Discipline", Annals of Applied Probability, No. 11, 2001.

[10] W. T. Chan, T. W Lam, K. S. Liu, P. W. H. Wong, "Resource augmentation analysis of SRPT and SJF for minimizing total stretch in multiprocessor scheduling", University of Liverpool, UK.

[11] L. Sha, R. Rajkumar, and S. S. Sathaye, "Generalized Rate-Monotonic Scheduling Theory: A Framework for 
Developing Real-Time Systems", Proceedings of the IEEE, Jan. 1994.

[12] G. Buttazzo, M. Spuri, F. Sensini, "Value vs. Deadline Scheduling in Overload Conditions", Proceedings of the th

16 IEEE Real-Time Systems Symposium (RTSS 1995), Pisa, Italy, pp. 90-99, December 5-7, 1995.

[13] S. Baskiyar, N. Meghanathan, "A Survey of Contemporary Real-Time Operating Systems", Informatica 29 (2005) 233-240.

[14] IEEE Information Technology - Portable Operating System Interface (POSIX): IEEE/ANSI Std 1003.1, 1996 Edition.

[15] S. Agrawal, P. Bhatt, K.K Shukla, "Modified MUF and EDF Algorithms for Overload Soft Real Time", WSEAS Conferences on Recent Advances in Systems, Communications and Computers, April 6-8 2008.
[16] J. H. Anderson, V. Bud, U. C. Devi, "An EDF-based Scheduling Algorithm for Multiprocessor Soft RealTime Systems", $17^{\text {th }}$ Euromicro Conference on Real-Time Systems, 2005. Trans. Roy. Soc. London, vol. A247, pp. 529-551, April 1955.

[17] G Buttazzo, Research Trends in Real Time Computing for Embedded Systems, 2006

[18] F. Balarin, L. Lavagno, P. Murthy, and A. S. Vincentelli, "Scheduling for Embedded Real-Time Systems", IEEE Design \& Test of Computer, January-March, 1998.

[19] N. C. Audsley, A. Burns, M. F. Richardson, A. J. Wellings "Hard Real-Time Scheduling: The DeadlineMonotonic Approach (1991)", Proceedings 8th IEEE Workshop on Real-Time Operating Systems and Software

[20] P. Brucker, "Scheduling Algorithms", Third Edition, Springer, 2001 